\title{
Umbilicated Hairy Auricular Mass Mimicking Accessory Tragus
}

\author{
Jeong Hwan Choi \\ Department of Otorhinolaryngology-Head and Neck Surgery, Sanggye Paik Hospital, Inje University College of Medicine, Seoul, Korea
}

Received July 12, 2019

Revised August 7,2019

Accepted August 29, 2019

Address for correspondence Jeong Hwan Choi, MD

Department of Otorhinolaryngology-

Head and Neck Surgery,

Sanggye Paik Hospital,

Inje University College of Medicine,

1342 Dongil-ro, Nowon-gu,

Seoul 01757, Korea

Tel $+82-2-950-1104$

Fax +82-2-935-6220

E-mail choijh92@paik.ac.kr
Trichofolliculoma (TF) is a follicular hamartoma in which hairs protruding out of single orifice. To the best of my knowledge, only two auricular TF has been reported in the English literature. Moreover, clinically TF have been described to mimic malignancy. I present a case of an adult female with mass at the intertragal notch of the left auricle for several years. The clinical diagnosis was thought to be epidermoid cyst, accessory tragus, and other benign skin adnexal tumor. To prevent recurrence, the wide local excision of the mass was performed. The final diagnosis of TF was made. No recurrence was noted during the follow-up of 1 year. It is important for otologists to be familiar with the clinical and pathologic characterization of TF, to make the correct diagnosis.

J Audiol Otol 2020;24(2):99-102

\section{Introduction}

Trichofolliculomas (TFs) are rare adnexal tumors, reported as hamartomatous tumors or as benign follicular neoplasms, commonly affecting the head and neck regions. However, auricular involvement is extremely rare, and to the author's best knowledge, only 2 cases of ear or ear canal TF has been described in the English literature [1,2]. These benign hair follicle-derived tumors may clinically mimic malignancy such as basal cell carcinoma of the auricle. Histopathological examination is most often required to confirm the diagnosis. Complete excision of the tumor is the treatment of choice, as remnants of the lesion can lead to recurrence despite its benign nature [3]. Herein, the author describes a rare case of a TF arising from the auricle and review the relevant literature.

\section{Case Report}

A 48-year-old female patient presented with a several-year history of a slowly increasing asymptomatic mass in the lateral surface of the left auricle, near the concha. The patient could

This is an Open Access article distributed under the terms of the Creative Commons Attribution Non-Commercial License (https://creativecommons.org/licenses/by-nc/4.0/) which permits unrestricted non-commercial use, distribution, and reproduction in any medium, provided the original work is properly cited. not recall any history of trauma at the site of swelling. The lesion appeared as a solitary $0.8 \mathrm{~cm}$ skin-colored, dome-shaped nodule with irregular borders. In detail, the round mass had central small pitted umbilication and a tuft of fine hair protruding out of it (Fig. 1). In palpation, it was smooth, rubbery to firm in consistency, and fixed to the the intertragal notch of the left auricle without cartilage component. The remainder of the physical examination was unremarkable. There were no local lymphadenopathy and any other masses in the body in physical examination. Clinical diagnosis of accessory tragus, hair follicle nevus, other adnexal tumor, and basal cell carcinoma were also made due to irregular surface.

Because the patient wanted to remove the mass, wide local excision was planned. The mass was removed completely. Intraoperatively, it was adherent to the auricular epidermis so that it was inevitable to expose the auricular perichondrium. After removal of the mass, the exposed wound and perichondrium was covered with split-thickness skin graft. The biopsied tissue was single, firm, globular grey white and measured $0.6 \times 0.5 \times$ $0.5 \mathrm{~cm}$ in size.

Histopathological examination showed a dilated central cystic follicle that communicated with the overlying skin surface. The dilated follicular opening showed keratin debris and hair. The follicle was lined with squamous epithelium and surrounded by numerous smaller secondary follicles, many of 
which contained fragments of hair shafts along with many variables sized hair follicles exhibiting varying degree of maturation (Fig. 2). Epidermis and dermis unremarkably showed strands of stratified squamous epithelium. Many fibroblasts was observed at the the stroma. The histopathological analysis was consistent with TF. There was no recurrence after a follow up of 1 year.

\section{Discussion}

The TF is a rare adnexal hamartoma of the skin with differentiation to hair follicles [4]. The TF is found mostly in adults as a fresh-colored solitary nodule at the face and scalp [4]. Rarely, the external auditory canal, intranasal area, lip, and vulva are affected [5]. No definitive racial and sexual predilection is reported.

The TF is clinically characterized by a small, asymptomatic, skin colored dome shaped nodule with a central pore, through which a small tuft of one or two hairs protrude. Sometimes, the pore of the nodule appears as a black spot. If the hair has been plucked or is too fine and ignored during examination, the growth may mimic basal cell carcinoma or molluscum contagiosum. Furthermore, if a dilated central cystic follicle is missed on histological sections, and the secondary follicles fail to show any hair structures, then the lesion may misdiagnosed as a trichoepithelioma or even basal cell carcinoma.

TF was first described by Miescher [6] in 1944 as a nodule with a central large pore from which a tuft of hairs protruding. In 1957, Hyman and Clayman [7] reported a similar case and labeled it a "hair follicle nevus." However, Kligman and Pinkus [8] suggested the term "folliculoma," as it differs from a nevus by the presence of hair follicle.

The etiology of TF is uncertain, onset is usually spontaneous. However, history of previous trauma has been reported [9]. TF is cosmetically disfiguring and patients may tend to pluck out the hair emerging from it, which sometimes may lead to an inflammatory reaction. There are no any reports for relating systemic or skin disorders and they are considered as an abortive differentiation of pluripotent skin cells toward hair follicles.
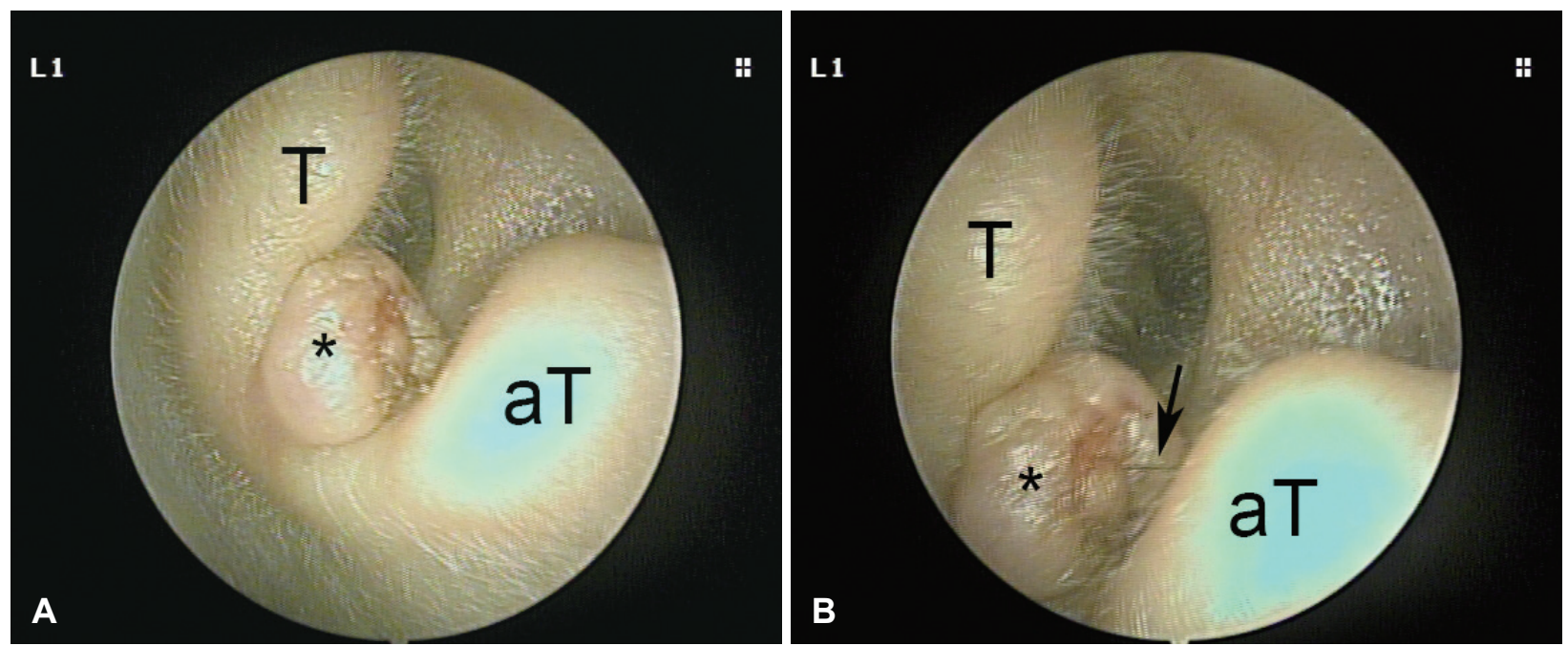

Fig. 1. (A) Clinical image showing an $8 \mathrm{~mm}$ dome skin-colored, dome shaped nodule (asterisk) with irregular borders. (B) It is fixed to the intertragal notch of the left auricle. Magnified clinical photograph showing a tuft of hair (arrow) arising above the central pit of nodule (asterisk). T: tragus, aT: antitragus.
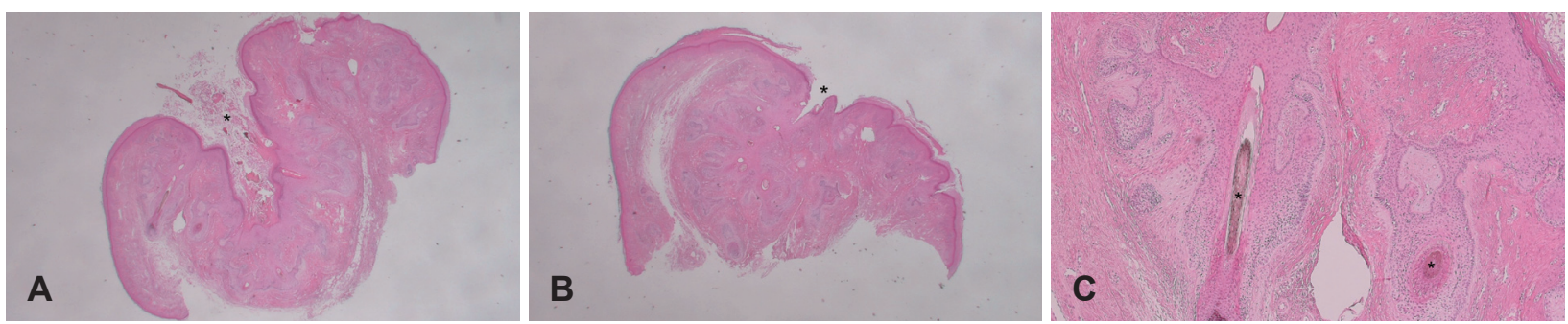

Fig. 2. (A) Keratin-filled, infundibular cyst (asterisk) which is lined by squamous epithelium, is dilated exceedingly with connection to skin surface with abnormal secondary hair follicles radiating from it (hematoxylin and eosin; original magnification $\times 10$ ). (B) Histologic examination demonstrates primary infundibular cystic structure (asterisk) in connection with the overlying epidermis with secondary, and tertiary follicles emanating from primary follicles (hematoxylin and eosin; original magnification $\times 10$ ). (C) Higher magnification reveals the cystic structure with the terminal hair shaft (asterisks) (hematoxylin and eosin; original magnification $\times 100$ ). 
According to Kligman and Pinkus [8], it shows intermediate differentiation between a hair follicle nevus, which is simple hyperplasia of the hair follicle, and a trichoepithelioma, which usually lacks a mature hair follicle. It might be also misdiagnosed as sebaceous cyst, nevus, molluscum contagiosum, or even basal cell carcinoma, especially when the hair has been missed [9].

Histopathological examination is mandatory to confirm the diagnosis. Definite clue is a stratified squamous epithelium lined cystic follicle that communicated with the overlying skin surface. Many secondary follicles can be observed around the central cystic follicle. Scanty lymphocytic or graulomatous inflammation might be observed. Within the follicular epithelium, many merkel cells have been present, supporting that TFs are hamartomas. In addition, it can be subdivided into three stages, which are early, fully developed, and late stage [10]. In early stages, there are few secondary hair follicles originating from a primary one. In very early stages, it may mimic trichoepithelioma, dermal nevus, or epidermoid cyst if the pore is not clearly visible. In mature lesions, vellus hair follicles increase in number. Later stages show a thickened primary follicle and fewer secondary follicles [9]. The evolution of TF is considered similar to a regressing normal hair follicle but probably in a one way direction with no regeneration from telogen to anagen.

$\mathrm{TF}$ is considered a benign entity, despite one case report of malignant transformation with perineural invasion [11]. Local excisional biopsy is diagnostic and further treatment may not be needed.

Histologically, hair follicle nevus and accessory tragi are the most difficult to distinguish of the lesions in the differential diagnosis of TFs. Their histologic backgrounds are very similar except the amounts of hair follicles and subcutaneous fat [12]. $\mathrm{TF}$ is a pilar tumor and intermediate in differentiation between a trichoepithelioma, which misses maturation of the hair follicle and a hair follicle nevus, which is simple hyperplasia of the hair follicle. On the other hand, central cyst and radiating hair follicles are much found in the TF.

Accessory tragus is a more complex structure, which consists not only of a pilar complex but also cutaneous appendages in different stages of development. A central cartilage structure is present in most accessory tragi. Many authors have stated that accessory tragi and hair follicle nevi are on the same spectrum. Asahina, et al. [13] stated that, although this may be the case, the developmental mechanisms might vary according to the location or component of the lesion.

Satoh, et al. [14] reported the accessory tragi had a prominent connective tissue in subcutaneous fat. In contrast, Ban, et al. [15] explained that a connective tissue framework in subcutaneous fat appears in both hair follicle nevus and accesso- ry auricle, and that the accessory auricle can be differentiated by the presence of abundant, subcutaneous fat.

When a lesion located in a preauricular area at birth or when it contains cartilage part, we may call it accessory tragus. In contrast, a lesion occurs in an atypical area for accessory tragus, and miss cartilage and is then interpreted as hair follicle nevus.

Treatment of TF is usually not required. At times, TF may be disfiguring, and the pulling away of hairs and other injuries may cause inflammatory reactions requiring treatment. In such cases and for the confirmative diagnosis, surgical excision is usually inevitable and recommendable. The prognosis is excellent, although recurrence can occur at the primary site rarely [3].

In conclusion, TF is a benign nodular hamartoma, rarely involving the auricle. Although rare, it should be considered in the differential diagnosis in all cases of asymptomatic, fleshcolored, dome shaped, solitary auricular nodule with a central pit and tufts of hairs. Complete excision of the tumor is the treatment of choice, and incomplete excision may lead to recurrence.

\section{Conflicts of interest}

The author has no financial conflicts of interest.

\section{ORCID iD}

Jeong Hwan Choi

https://orcid.org/0000-0001-7348-9861

\section{REFERENCES}

1) O’Mahony JJ. Trichofolliculoma of the external auditory meatus. Report of a case and a review of the literature. J Laryngol Otol 1981; 95:623-5.

2) Srivastava RN, Ajwani KD. Trichofolliculoma. Ear Nose Throat J 1979;58:159-60.

3) Ghosh SK, Bandyopadhyay D, Barma KD. Perifollicular nodule on the face of a young man. Indian J Dermatol Venereol Leprol 2011; 77:531-3.

4) Gray HR, Helwig EB. Trichofolliculoma. Arch Dermatol 1962;86: 619-25.

5) Choi CM, Lew BL, Sim WY. Multiple trichofolliculomas on unusual sites: a case report and review of the literature. Int J Dermatol 2013;52: 87-9.

6) Miescher G. Trichofolliculoma. Dermatologica 1944;89:193-4.

7) Hyman AB, Clayman SJ. Hair-follicle nevus; report of a case and a review of the literature concerning this lesion and some related conditions. AMA Arch Derm 1957;75:678-84.

8) Kligman AM, Pinkus H. The histogenesis of nevoid tumors of the skin: The folliculoma--a hair-follicle tumor. AMA Dermatol 1960; 81:922-30.

9) Singh N, Kumar N, Chandrashekar L, Thappa DM, Kar R, Srinivas BH. Umbilicated nodule over eyebrow. Dermatol Online J 2013;19: 19622.

10) Schulz T, Hartschuh W. The trichofolliculoma undergoes changes corresponding to the regressing normal hair follicle in its cycle. $\mathrm{J}$ Cutan Pathol 1998;25:341-53.

11) Stern JB, Stout DA. Trichofolliculoma showing perineural invasion. 
Trichofolliculocarcinoma? Arch Dermatol 1979;115:1003-4.

12) Karabulut YY, Şenel E, Karabulut HH, Dölek Y. Three different clinical faces of the same histopathological entity: hair follicle nevus, trichofolliculoma and accessory tragus. An Bras Dermatol 2015; 90:519-22.

13) Asahina A, Mitomi H, Sakurai N, Fujita H. Multiple accessory tragi without cartilage: relationship with hair follicle naevi? Acta Derm
Venereol 2009;89:316-7.

14) Satoh T, Tokura Y, Katsumata M, Sonoda T, Takigawa M. Histological diagnostic criteria for accessory tragi. J Cutan Pathol 1990;17: 206-10.

15) Ban M, Kamiya H, Yamada T, Kitajima Y. Hair follicle nevi and accessory tragi: variable quantity of adipose tissue in connective tissue framework. Pediatr Dermatol 1997;14:433-6. 